\title{
Numerical study of convective heat transfer of a supersonic combustor with varied inlet flow conditions
}

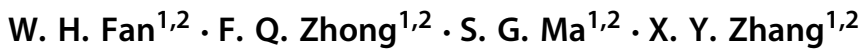

Received: 4 April 2019 / Revised: 20 May 2019 / Accepted: 27 May 2019 / Published online: 21 June 2019

(C) The Chinese Society of Theoretical and Applied Mechanics and Springer-Verlag GmbH Germany, part of Springer Nature 2019

\begin{abstract}
Characteristics of convective heat transfer of a supersonic model combustor with variable inlet flow conditions were studied by numerical simulation in this paper. The three-dimensional flow and wall heat flux at different air inlet Mach numbers of 2.2, 2.8 and 3.2 were studied numerically with Reynolds-averaged Navier-Stokes equations with a shear-stress transport (SST) $k$ $-\omega$ turbulence model and a three-step reaction model. Meanwhile, ethylene was chosen as the fuel, and the fixed fuel-to-air equivalence ratio is 0.8 in all cases in this paper. The results of the simulations indicate that wall heat flux distribution of the combustor is very non-uniform with several peaks of wall heat flux at varied locations. For the low inlet Mach number of 2.2, a shock train structure is formed in the isolator, and three peaks of wall heat flux are located respectively on the backward face of the cavity, on the side wall near the fuel injection and on the bottom wall near the injection holes, and a maximum wall heat flux reaches $5.4 \mathrm{MW} / \mathrm{m}^{2}$. For the medium inlet Mach number of 2.8, there exists a much shorter shock structure with three peaks of wall heat flux similar to that of Mach number 2.2. However, as the inlet Mach number increased to 3.2, there is no shock structure upstream of fuel injections, and the combustor flow is in a supersonic mode with different locations and values of wall heat flux peaks. The statistical results of wall heat loading show that the change of total wall heat is not monotonic with the increase of inlet Mach number, and the maximum appears in the case of Mach number being 2.8. Meanwhile, for all the cases, the bottom wall takes up more than $50 \%$ of the total heat loading.
\end{abstract}

Keywords Wall heat flux $\cdot$ Numerical simulation $\cdot$ Ethylene $\cdot$ Supersonic combustor

\section{Introduction}

The thermal environment of a supersonic combustor is very severe due to significant aerodynamic heating and release of combustion heat. Therefore, thermal protection of the combustor is one of the key technologies for scramjet applications. Finding ways to measure and predict the wall heat flux of a supersonic combustor is becoming one of the focused issues.

The measurement of heat flux of the inner wall of a supersonic combustor includes two major ways of direct and indirect methods. The direct measuring method often

$\triangle$ F. Q. Zhong

fzhong@imech.ac.cn

1 State Key Laboratory of High Temperature Gas Dynamics, Institute of Mechanics, Chinese Academy of Sciences, Beijing 100190, China

2 School of Engineering Science, University of Chinese Academy of Sciences, Beijing 100049, China uses a high-temperature heat flux gage [1] or surface junction thermocouple sensor [2] to directly measure wall heat flux. Indirect measuring methods such as the conjugate gradient method based on the functional principle used by Cheng et al. [3] or a data processing method based on variables separation researched by Sun et al. [4] have been developed and used for measurements of inner wall heat flux of a combustor. However, the present measurement methods can obtain wall heat fluxes of only a few points on the combustor wall with relatively poor spatial resolutions. Except for the experiment for measuring heat flux, some research on fluid flow has been presented as well. Further investigation was conducted by Wang et al. [5] on the flow characteristics of cavity-assisted hydrogen jet combustion in a supersonic flow by optical diagnosis-based combustion experiments.

Compared to the experimental method, numerical methods can obtain wall heat flux distributions of the whole combustor rather than several points, and provide more details of flow fields. So far there have been many researchers using numerical simulation to study characteristics of combustion 


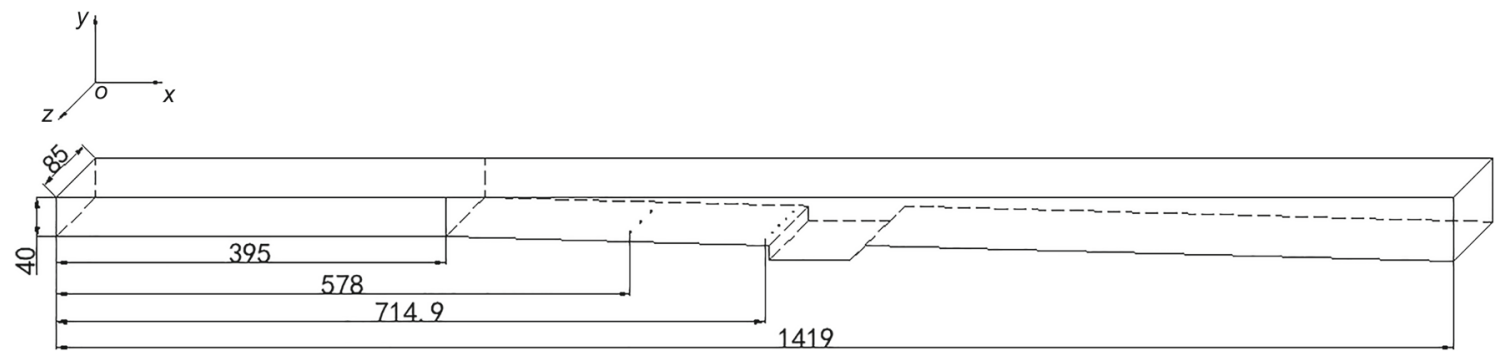

Fig. 1 Schematic diagram of the model combustor (unit: $\mathrm{mm}$ )

flow and heat transfer. For example, Ai et al. [6] used the Monte Carlo method to calculate radiative heat transfer in a scramjet combustor and analyzed how the hot gas heats combustor walls in a radiant way. Subsequently, Jiang et al. [7] researched the effect of nozzle geometrical and flow parameters on heat loading of a liquid-propellant rocket engine with the Reynolds-averaged Navier-Stokes (RANS) method. A hydrogen-fueled scramjet combustor with a dual cavity was investigated by Wang et al. [8] using large eddy simulation, and the recycling/rescaling method was adopted to generate unsteady turbulent inflow conditions in their research. Except for the simulation research, there is some literature focusing on the numerical methods for more accurate calculation. For example, Lv and Ihme [9] researched the high-order discontinuous Galerkin method for simulations of multicomponent and chemically reacting flows. Further study by Zhao et al. [10] focused on an efficient unstructured weighted essentially non-oscillatory (WENO) method that is perfectly appropriated for simulation of supersonic reactive flows.

Recently, Wang et al. [11] used the RANS method with the shear-stress transport (SST) $k-\omega$ model to simulate combustion and heat transfer of a supersonic combustor with an inlet Mach number of 2.5, and identified locations of peaks of wall heat flux. However, the work of Ref. [11] studying the heat transfer of a supersonic combustor utilized only one particular inlet flow condition. For realistic combustor operations, the inlet conditions of a supersonic combustor always change due to the variation in flight conditions such as flight Mach number. To the authors' knowledge, so far, a systematic study on the properties of wall heat flux of a supersonic combustor with variable inlet conditions has not been reported yet.

In this paper, a supersonic model combustor with ethylene fuel is numerically studied at varied inlet conditions with typical Mach numbers. RANS equations are solved with the SST $k-\omega$ turbulence model and a three-step reaction model for ethylene. The present paper is aimed to provide some insights to identify and understand combustion flow and distributions of wall heat flux of a supersonic combustor.

\section{Numerical method}

\subsection{Configuration and boundary conditions}

Figure 1 is the schematic diagram of the model combustor. The overall length of the combustor is $1419 \mathrm{~mm}$ ( $x$-direction), the height and width of the inlet cross section are $40 \mathrm{~mm}$ and $85 \mathrm{~mm}$, respectively. The combustor has a constant cross section isolator with length of $395 \mathrm{~mm}$ and two expanded sections with divergent angles of $1.5^{\circ}$ and $2^{\circ}$, as well as a cavity with length of $80 \mathrm{~mm}$ and the length-to-depth ratio of 5.5. There are two series of injections on the bottom wall as shown in the Fig. 1; the upstream injections are the main fuel injections with hole diameter of $2 \mathrm{~mm}$, and the downstream ones are the minor fuel injections with hole diameter of $0.8 \mathrm{~mm}$. Ethylene is used as fuel and the total fuel equivalence ratio is 0.8 for all the cases, but the fuel equivalence ratios of the two series of injections are variable. The combustor has bilateral symmetry, thus only half of the combustor was applied as the computational domain in this study, and the total grids number is 3.3 million. The grids near the wall are refined, and the first grid point from the wall is at $\Delta y^{+} \sim 1$.

Three cases with varied inlet Mach number and inlet temperature, pressure for typical working conditions of supersonic combustor are studied in this paper. The inlet Mach numbers for cases 1, 2 and 3 are 2.2, 2.8 and 3.2 with inlet total temperature of $1400 \mathrm{~K}, 1900 \mathrm{~K}$ and $2200 \mathrm{~K}$ and inlet static pressure of $90 \mathrm{kPa}, 63 \mathrm{kPa}$ and $50 \mathrm{kPa}$, respectively.

The boundary condition of the air inlet is pressure inlet condition with pre-given total temperature and pressures. The turbulent intensity at the inlet is set as $1 \%$. For fuel injection, the fuel pressure and temperature are given with an injection Mach number of unity. The boundary condition of the combustor outlet is pressure outlet for supersonic flow. The wall boundary condition is no-slip condition with a constant temperature of $1000 \mathrm{~K}$. 


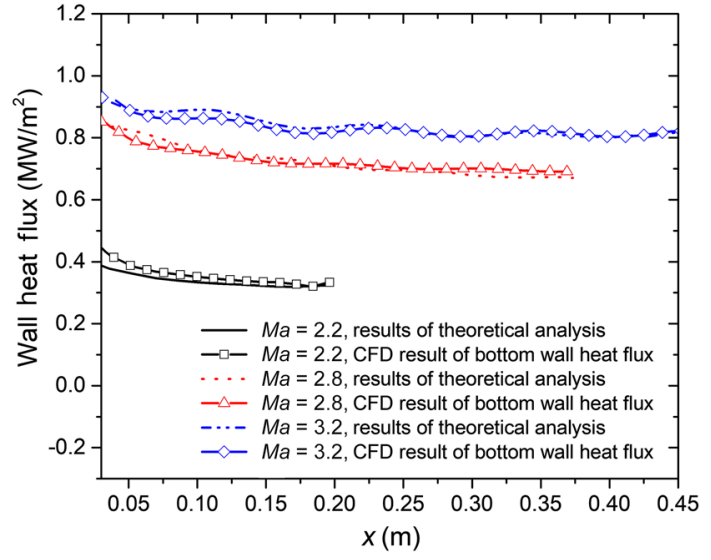

Fig. 2 Comparison of wall heat flux by computation and by the theoretical method of Eckert

\subsection{Numerical methods and validations}

Favre-averaged Navier-Stokes equations are solved with the SST $k-\omega$ turbulence model with compressibility and low Reynolds number corrections [12]. Besides, the advection upstream splitting method (AUSM) [13] with a second-order upwind difference scheme is used to calculate the convective terms, and a second-order central scheme is for the viscous term. A three-step reaction model of ethylene proposed by Westbrook and Dryer [14] is used, and a combination of the finite-rate model and eddy-dissipation model (FR/EDM model) $[15,16]$ is applied to simulate the interaction process of reactions and turbulence. Numerical validation that compares the simulation results with the experimental data of wall pressures for fuel-to-air equivalence ratio of 0.46 and 0.9, and then an inlet Mach number of 2.5, has been made and reported in our previous work [11] with the same numerical methods and reaction model as those of this paper. The validation shows that the numerical results agree well with the experimental data, indicating good accuracy of the present simulation. Furthermore, the Eckert reference enthalpy method [17] is used in this paper to give a theoretical result of wall heat flux for the validation. The isolator part of the combustor where there is no combustion and shocks is characterized by the supersonic boundary layer, so that the distribution of wall heat flux in the isolator can be determined by the reference enthalpy method $[18,19]$ and used for the wall heat flux validation of the present numerical method. Figure 2 gives distributions of bottom wall heat flux obtained by the computation and the results by the theoretical method of Eckert [17] for the three cases. It is worth mentioning that the range of $x$ axis of each case is different, because there is shock structure or not in the isolator due to the downstream fuel combustion, and the length of the shock structure is different. Therefore, only the data in the isolator without shock structure is able to be used for the validation. As shown in Fig. 2, a good agreement is observed with a maximum discrepancy of less than $5 \%$.

\section{Results and discussion}

\subsection{Case 1 with an inlet Mach number of $\mathbf{2 . 2}$}

Figure 3 gives image of numerical schlieren at the $x-y$ planes in the center for case 1 with an inlet Mach number of 2.2. The schlieren image clearly shows the shock structures of the flow field. In Fig. 3, a shock train structure is formed upstream of the fuel injection to match the pressure difference between the inlet and the combustion region. Figure 4 plots contours

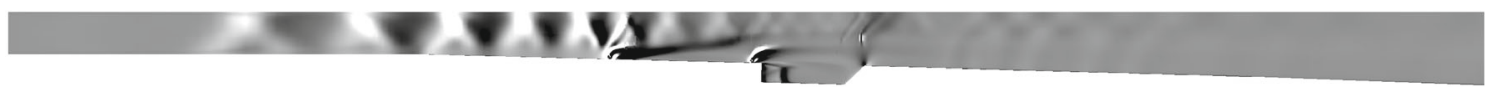

Fig. 3 Image of schlieren at the symmetry $x-y$ plane $(z=42.5 \mathrm{~mm})$

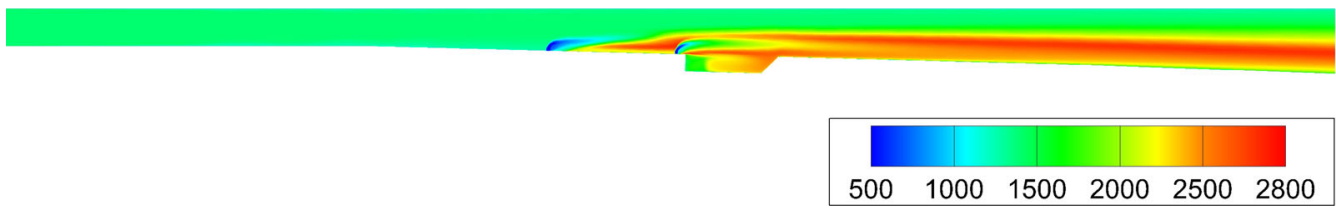

Fig. 4 Contours of total temperature at the symmetry $x-y$ plane $(z=42.5 \mathrm{~mm})$ (unit: K)

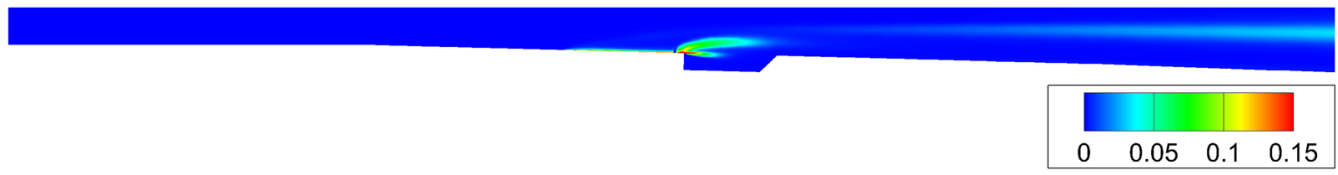

Fig. 5 Contours of $\mathrm{CO}_{2}$ mass fraction at the symmetry $x-y$ plane $(z=42.5 \mathrm{~mm})$ 
a
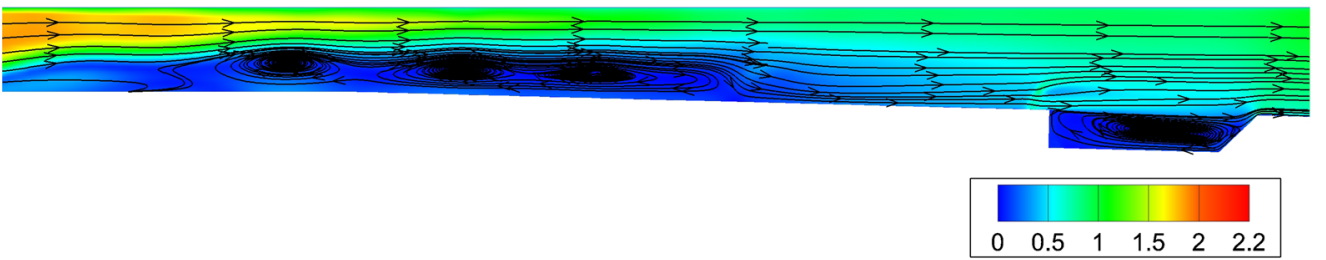

b

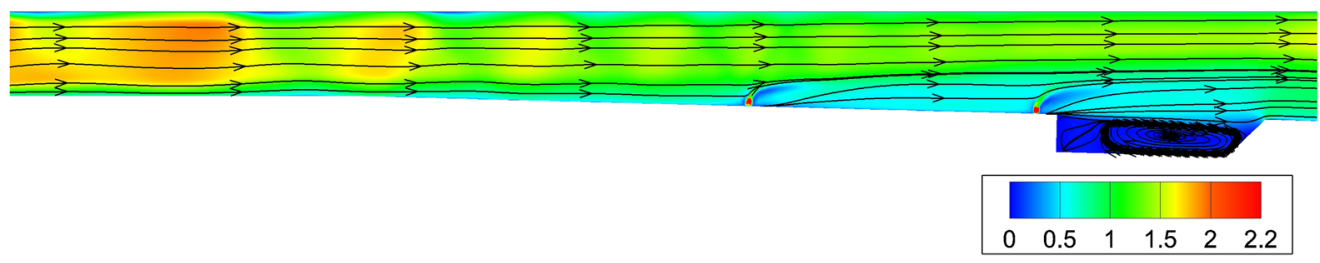

Fig. 6 Contours of Mach number and streamline distributions at different $x-y$ planes. a Near the side wall, $z=10 \mathrm{~mm}$. b At the symmetry plane, $z=42.5 \mathrm{~mm}$
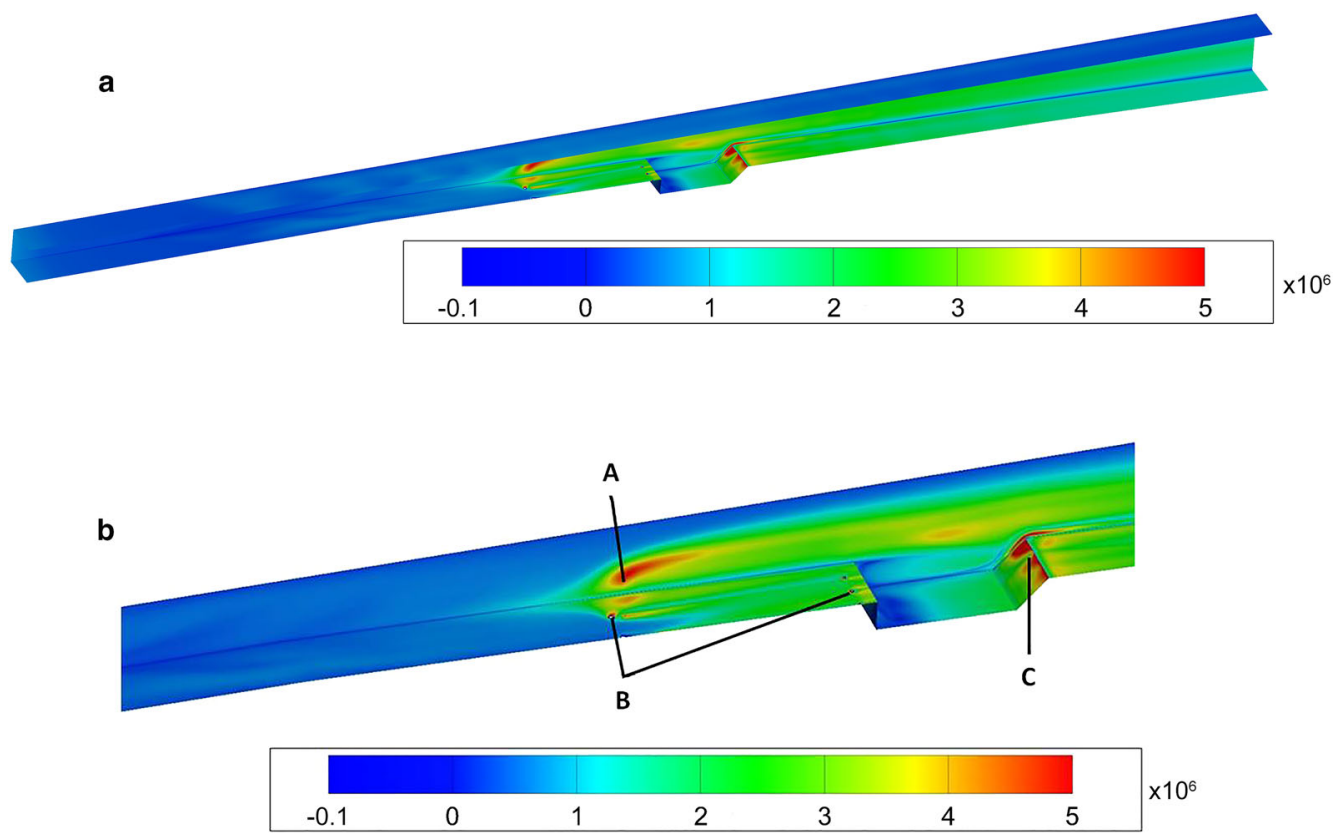

Fig. 7 Contours of wall heat flux. a Including the top wall. b Zoom-in of plot a with indication of heat flux peaks and excluding the top wall

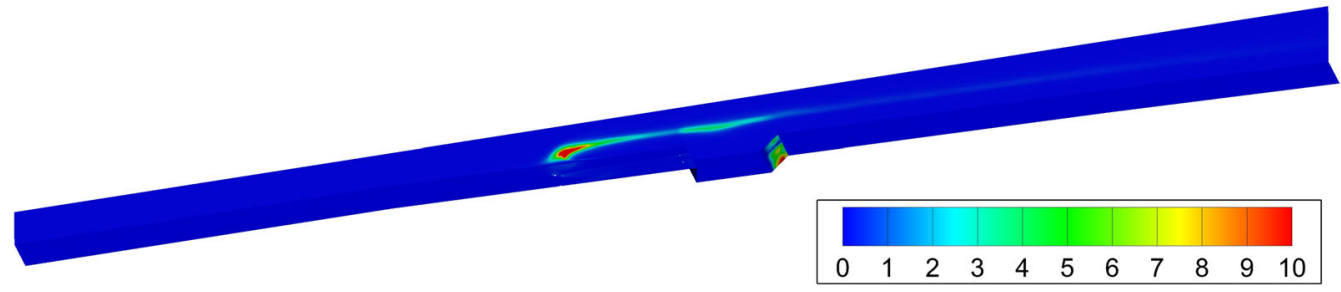

Fig. 8 Contours of the reaction rate for $\mathrm{CO} \rightarrow \mathrm{CO}_{2}$ on the side wall and the bottom wall (unit: $\mathrm{kmol} /\left(\mathrm{m}^{3} \cdot \mathrm{s}\right)$ )

of total temperature at the $x-y$ plane in the center. The hightemperature region is close to the bottom of the combustor and in the cavity due to the fuel injections at the bottom side.
There are obvious low-temperature regions just downstream of the injections because of spraying of the low-temperature ethylene. Contours of $\mathrm{CO}_{2}$ mass fraction at the $x-y$ plane 

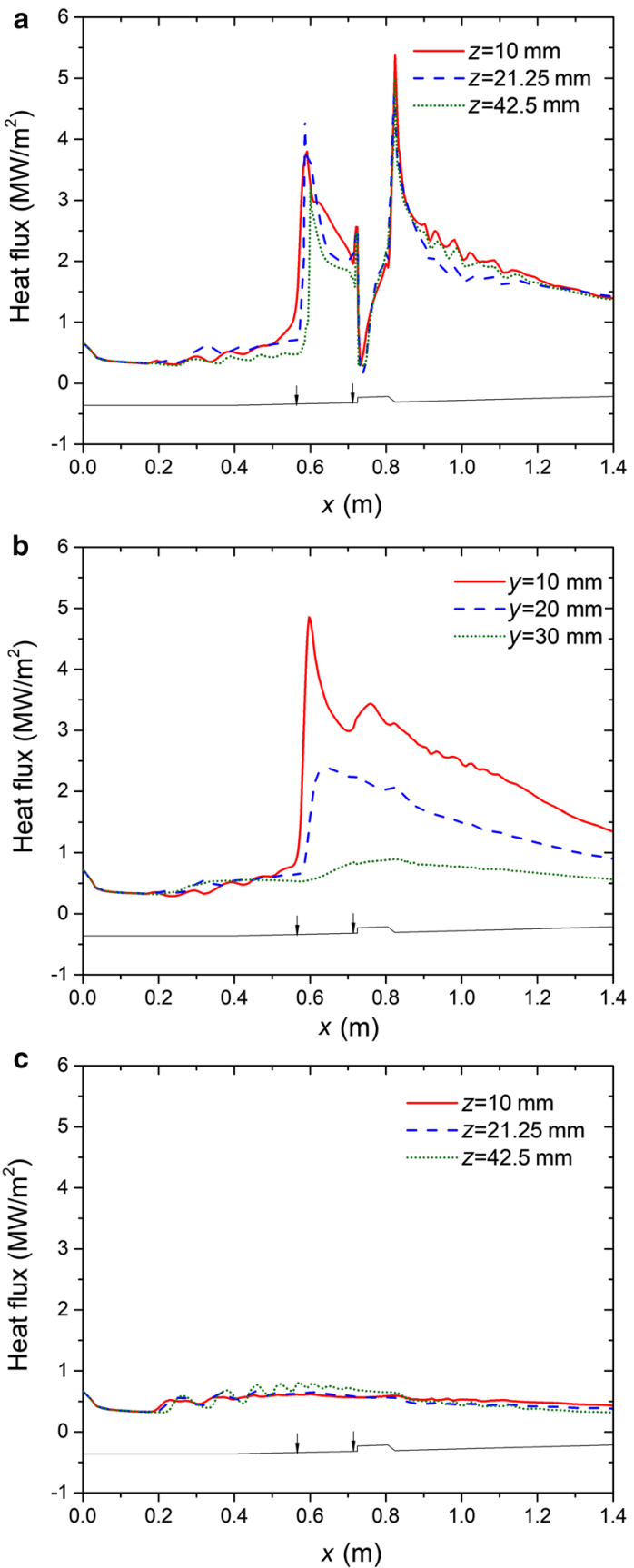

Fig. 9 Heat flux distribution curves on different walls of the combustor. a Bottom wall, b side wall, $\mathbf{c}$ top wall

in the center is shown in Fig. 5. As known, the formation reaction of $\mathrm{CO}_{2}$ is the main reaction for heat releasing, so that Fig. 5 is plotted to show the combustion situation. It is apparent that the distribution of $\mathrm{CO}_{2}$ mainly concentrates on the cavity shear layer and the downstream location near the combustor outlet, which indicates the major places for combustion to take place.
Contours of Mach number and streamline distribution at the $x-y$ planes near the side wall and in the middle are separately presented in Fig. 6a, b. Figure 6a indicates that there are obvious low-speed regions near the side wall since separation of the boundary layer takes place due to interactions between the local shock structure as shown in Fig. 3 and the boundary layer. Meanwhile, a low-speed region is also found in the cavity, as shown in both Fig. 6a and b. The existence of low-speed regions with vortex structures can make the fuel and air mix better and promotes combustion.

Figure $7 \mathrm{a}, \mathrm{b}$ gives the distribution of wall heat flux of different combustor walls. The heat flux distribution of the top wall is comparatively uniform with slight non-uniformity caused by the shock train structure as mentioned before. However, as shown in Fig. 7b, significant non-uniform distribution of heat flux and the local peak values appear on the bottom wall and the side wall. It is seen that a few heat flux peaks are identified, among which, for the bottom wall, two peaks (denoted as B and C) are respectively located near the injection holes and on the backward face of the cavity, and for the side wall, one peak (denoted as A) is located downstream of the main fuel injector. It is believed that the heat flux peaks are related to local flow and combustion properties, and the locations of heat flux peaks are all near the low-speed regions with vortices where fuel and air mixing is sufficient and combustion is intensive. Meanwhile, as known, the reaction with main heat releasing is the reaction that produces carbon dioxide. Combined with the contours of the reaction rate for $\mathrm{CO} \rightarrow \mathrm{CO}_{2}$ on the side wall and the bottom wall, as shown in Fig. 8, it is obvious that the regions with intensive production of $\mathrm{CO}_{2}$ are also accompanied with a mass of heat release reactions. Therefore, it shows why local peaks of heat flux are located on the side wall and on the backward face of the cavity as illustrated in Fig. 7b.

Figure $9 \mathrm{a}-\mathrm{c}$ plots the heat flux curves along the $x$ direction at different spanwise or vertical locations on the bottom, the side and the top walls, respectively. On the top and the bottom walls, three locations with varied $z$ values $(z=10 \mathrm{~mm}$, $21.25 \mathrm{~mm}, 42.5 \mathrm{~mm}$ ) are chosen. On the side wall, three locations with different vertical positions $(y=10 \mathrm{~mm}, 20 \mathrm{~mm}$, $30 \mathrm{~mm}$ ) are chosen as well. As shown in Fig. 9a, there exists two high-heat-flux regions on the bottom wall, and the maximum value reaches $5.4 \mathrm{MW} / \mathrm{m}^{2}$ located on the backward face of the cavity. At different spanwise locations, the heat flux does not change very much, indicating a relatively good uniformity of heat flux distribution on the bottom wall along the spanwise direction. On the side wall as shown in Fig. 9b, location of the maximum heat flux with a value of $4.8 \mathrm{MW} / \mathrm{m}^{2}$ moves upstream and close to the main fuel injections, and, overall, the heat flux on the side wall decreases remarkably with the increase of height.

Combining these two sets of graph (Figs. 7 and 9) for analysis, it can be concluded that there are three local high- 
Fig. 10 Image of schlieren at the symmetry $x-y$ plane $(z=42.5 \mathrm{~mm})$
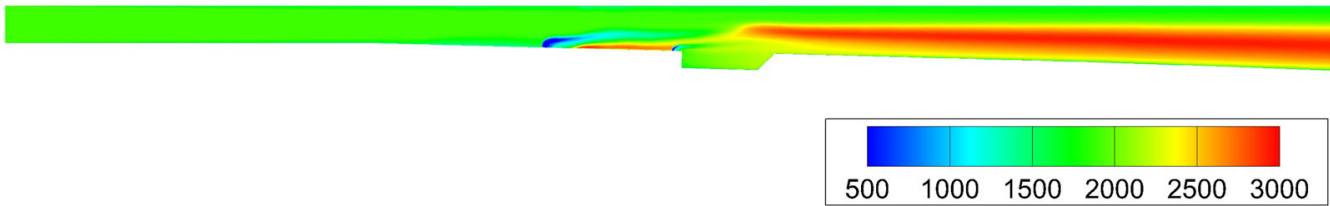

Fig. 11 Contours of total temperature at the symmetry $x-y$ plane $(z=42.5 \mathrm{~mm})$

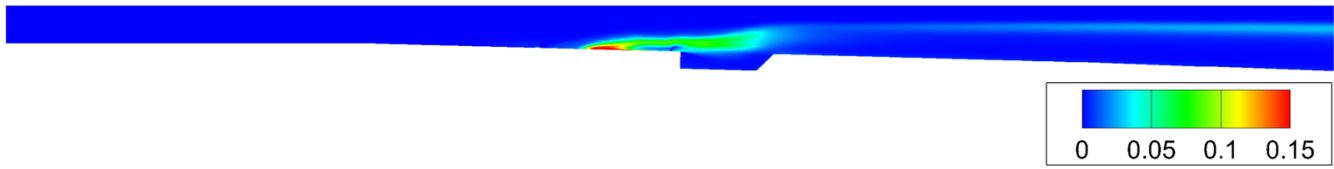

Fig. 12 Contours of $\mathrm{CO}_{2}$ mass fraction at the symmetry $x-y$ plane $(z=42.5 \mathrm{~mm})$

a

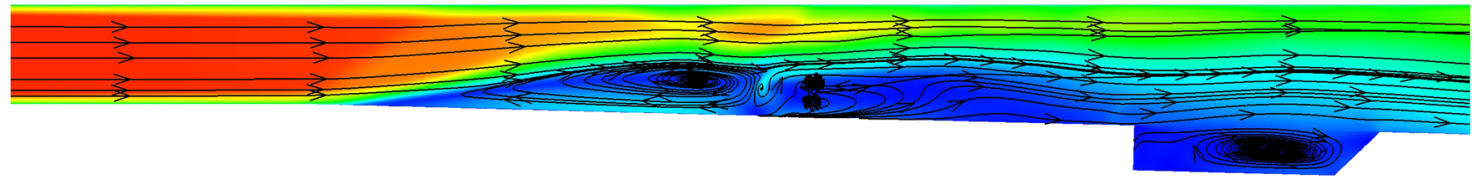

b

Fig. 13 Contours of Mach number and streamline distributions at different $x-y$ planes. a Near the side wall, $z=10 \mathrm{~mm}$. b At the symmetry plane, $z=42.5 \mathrm{~mm}$

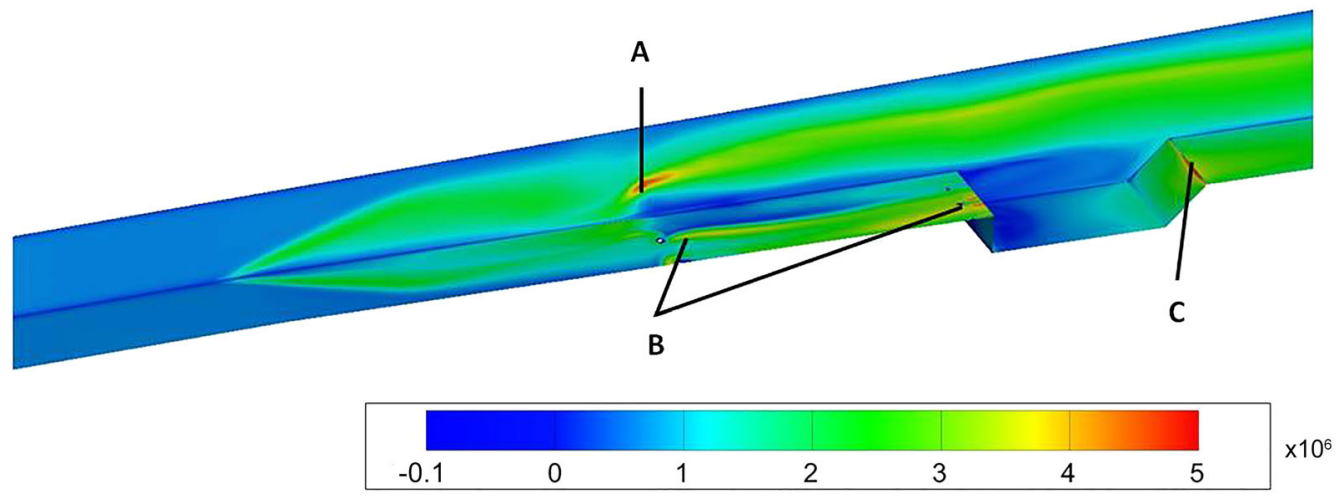

Fig. 14 Partially enlarged drawing of contours of wall heat flux

heat-flux regions on the combustor wall: (1) on the bottom wall around the injections; (2) on the side wall over the upstream injections; and (3) on the backward face of the cavity. Besides, Fig. 9 indicates that the maximum wall heat flux appears on the backward face of the combustor cavity, and the value reaches $5.4 \mathrm{MW} / \mathrm{m}^{2}$.

\subsection{Case 2 with an inlet Mach number of 2.8}

Figure 10 shows the image of numerical schlieren at the symmetry $x-y$ plane for case 2 , which indicates that an oblique shock train structure is generated in the isolator for case 2 . However, comparing with case 1 , the starting points of the 
shock train move backward, and the length of the shock structure becomes shorter with the increase of inlet Mach number. It is understandable that as the inlet Mach number increases, the pressure rise can be achieved across a shorter shock train structure.

Figure 11 presents the contours of total temperature at the $x-y$ plane in the middle for case 2 . The total temperature distribution in the middle of the combustor for case 2 as plotted in Fig. 11 is different to case 1 as shown in Fig. 4. On account of the higher velocity of air flow in case 2 , the fuel jets with low temperature move faster to the downstream, which causes a less effective mixing of fuel and air as well as combustion at the same downstream location compared to that of case 1 . Therefore, for case 2, the local high-temperature region appears in the downstream of the cavity rather than over the cavity like case 1 . Figure 12 gives the contours of $\mathrm{CO}_{2}$ mass fraction in the middle plane of the combustor for case 2. One can see that $\mathrm{CO}_{2}$ primarily distributes in the wake region of injection and the cavity shear layer. It is noted that some combustion occurs in the wake region of injection, which is caused by the intensive interaction of injection and incoming air flow with the faster velocity of incoming flow.

Figure 13a, b shows the contours of Mach number and streamline distribution near the side wall and in the middle of case 2 , such that there are low-speed regions with a vortex structure in the cavity and in the isolator. However, comparing with case 1, the low-speed region with flow separations in the isolator near the side wall becomes shorter.

Figure 14 shows the contours of wall heat flux distribution for case 2. As presented, the wall heat flux distribution of case 2 is analogous with the case 1 . In case 2 , there are three local high-heat-flux regions: (1) on the bottom wall around the injections; (2) on the side wall over the upstream injections; (3) on the backward face of the cavity. However, analyzing in combination with Fig. 15a-c which gives the distribution curves of heat flux, respectively, on the bottom wall, side wall and top wall, the values of the peaks and the areas of the local high-heat-flux regions are quite different for these two cases. For case 2 with a higher inlet Mach number, compared to those of case 1, it is found that both the heat flux peak C located on the backward face of the cavity and the peak A on the side wall become smaller. The maximum wall heat flux is on the side wall rather than on the bottom wall like case 1 , and the maximum value is $4.5 \mathrm{MW} / \mathrm{m}^{2}$ which is smaller than that of case 1 of $5.4 \mathrm{MW} / \mathrm{m}^{2}$. This is attributable to the much larger speed of air flow of case 2, which causes the fuel/air mixing and combustion to not be locally intensive as those of case 1 .

\subsection{Case 3 with an inlet Mach number of $\mathbf{3 . 2}$}

Figure 16 presents the numerical schlieren in the middle. Comparing with the cases in which inlet Mach numbers are
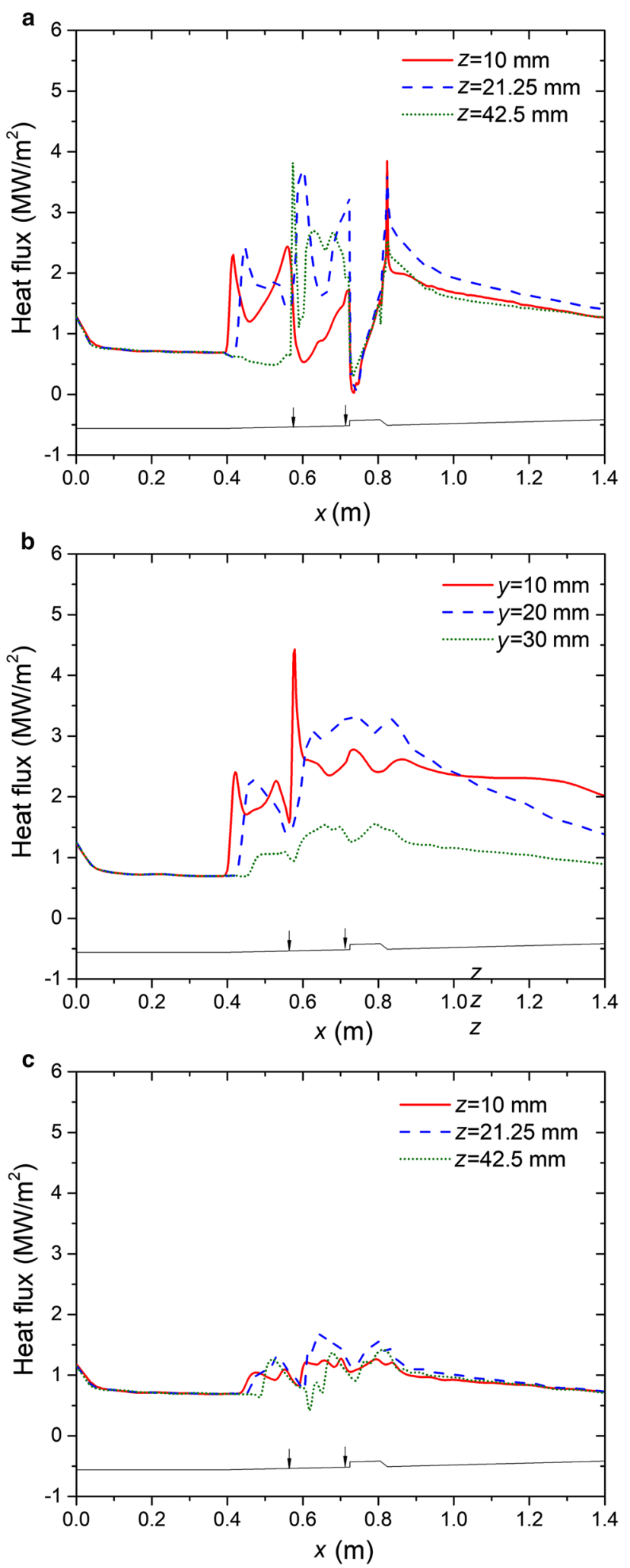

Fig. 15 Heat flux distribution curves on different walls of the combustor. a Bottom wall, $\mathbf{b}$ side wall, $\mathbf{c}$ top wall

2.2 and 2.8, there is no shock train structure in the isolator because of the increase of inlet Mach number, and the com- 


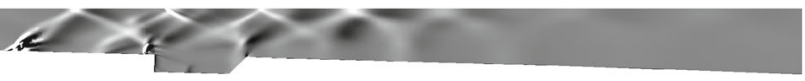

Fig. 16 Image of schlieren at the symmetry $x-y$ plane $(z=42.5 \mathrm{~mm})$
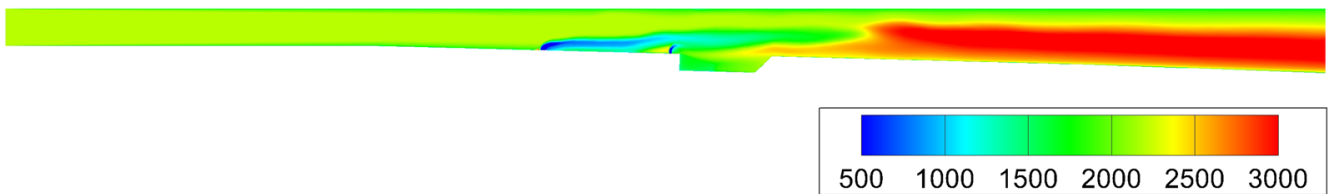

Fig. 17 Contours of total temperature at the symmetry $x-y$ plane $(z=42.5 \mathrm{~mm})$

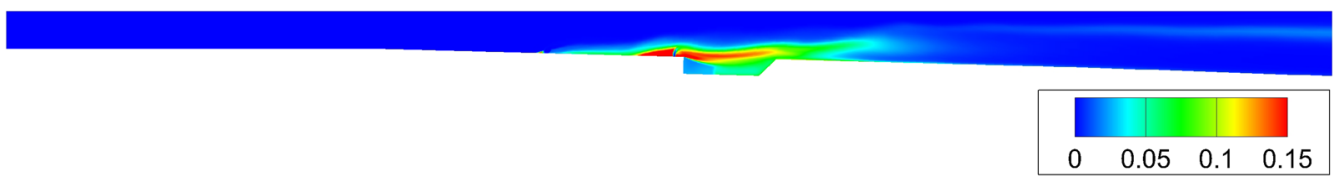

Fig. 18 Contours of $\mathrm{CO}_{2}$ mass fraction at the symmetry $x-y$ plane $(z=42.5 \mathrm{~mm})$

a

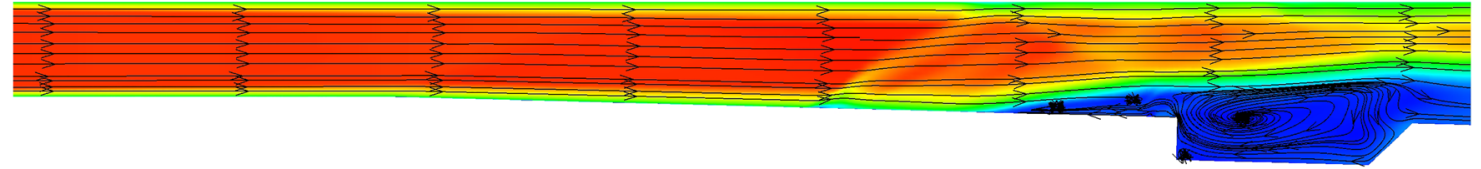

b

Fig. 19 Contours of Mach number and streamline distributions at the different $x-y$ planes. a Near the side wall, $z=10 \mathrm{~mm}$. b At the symmetry plane, $z=42.5 \mathrm{~mm}$

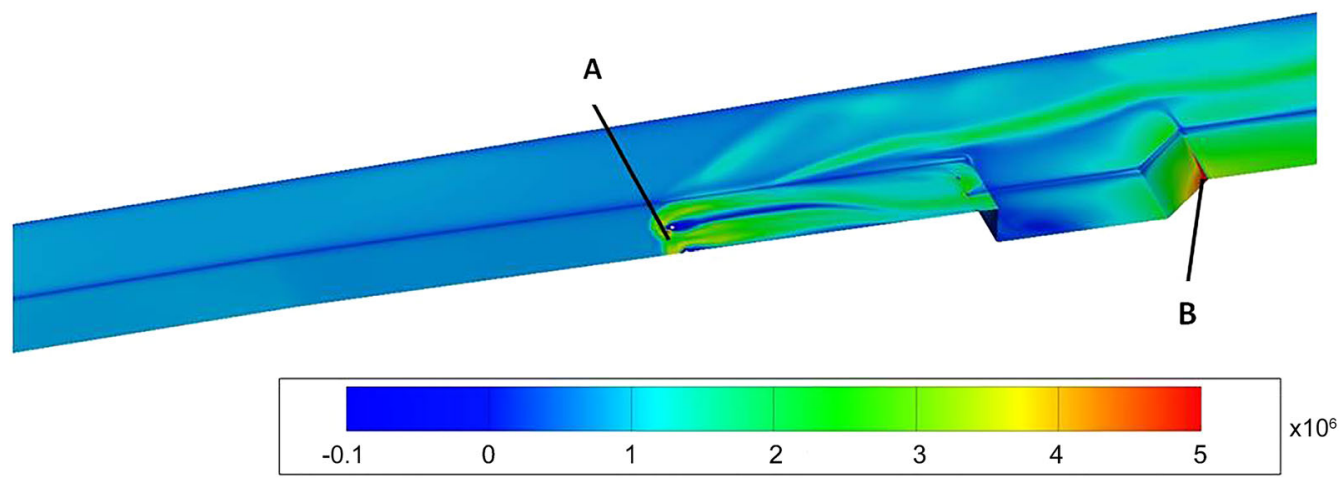

Fig. 20 Partially enlarged drawing of contours of wall heat flux of case 3

bustion of this case is a typical supersonic combustion mode [20]. Figure 17 shows the contours of total temperature in the middle. The distribution of total temperature presented in Fig. 17 is quite different from the last cases; the local hightemperature region in the middle $x-y$ plane is located in the downstream of the cavity instead of in the cavity, and a low- temperature region is observed above the cavity on account of the rapid movement of low-temperature fuel following the high-speed air flow. Figure 18 gives the contours of $\mathrm{CO}_{2}$ mass fraction in the middle. As is shown in the figure, $\mathrm{CO}_{2}$ mainly distributes in the wake region of injection and the cavity shear layer. However, the concentration of $\mathrm{CO}_{2}$ near 

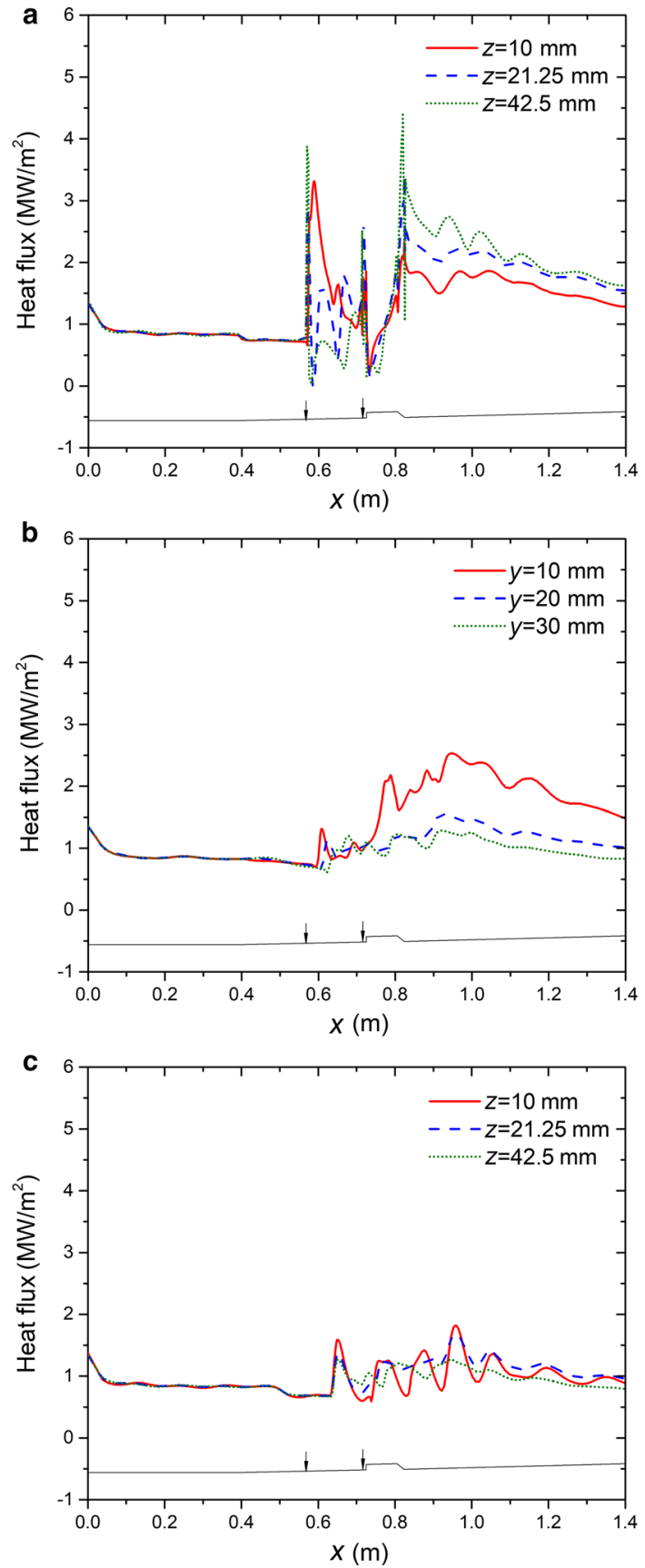

Fig. 21 Heat flux distribution curves on different walls of the combustor. a Bottom wall, b side wall, $\mathbf{c}$ top wall

the combustor outlet is not high, which may be on account of oxygen having been consumed in the upstream place, causing transformation of $\mathrm{CO}_{2}$ into $\mathrm{CO}$ and $\mathrm{O}_{2}$.

Figure $19 \mathrm{a}, \mathrm{b}$ gives the contours of Mach number and streamline distribution near the side wall and in the middle. Both figures show that there is no low-speed region and no large vortex structures in the isolator due to no generation of a shock train. Similar to the last cases, there is a stable low-speed region with a vortex in the cavity.

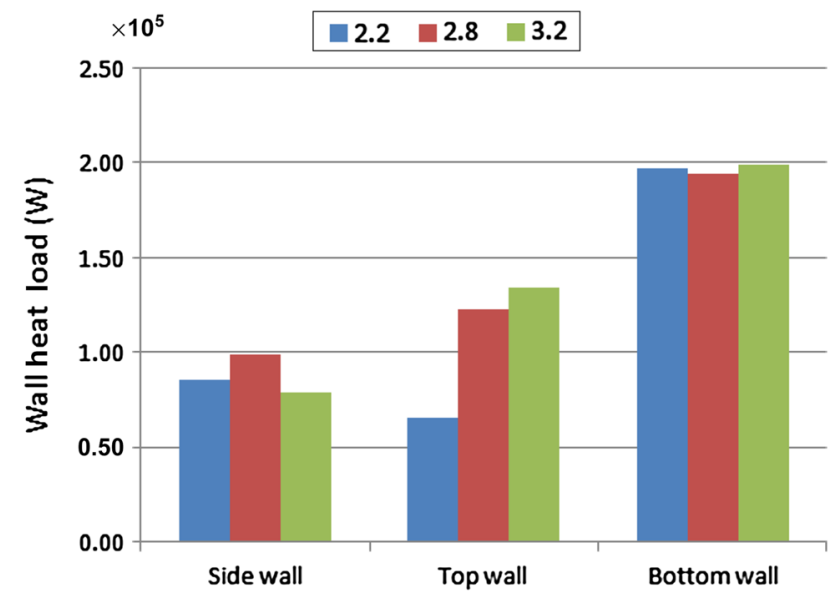

Fig. 22 Wall heat loading of different walls in different cases $(M a=$ 2.2, $M a=2.8, M a=3.2$ )

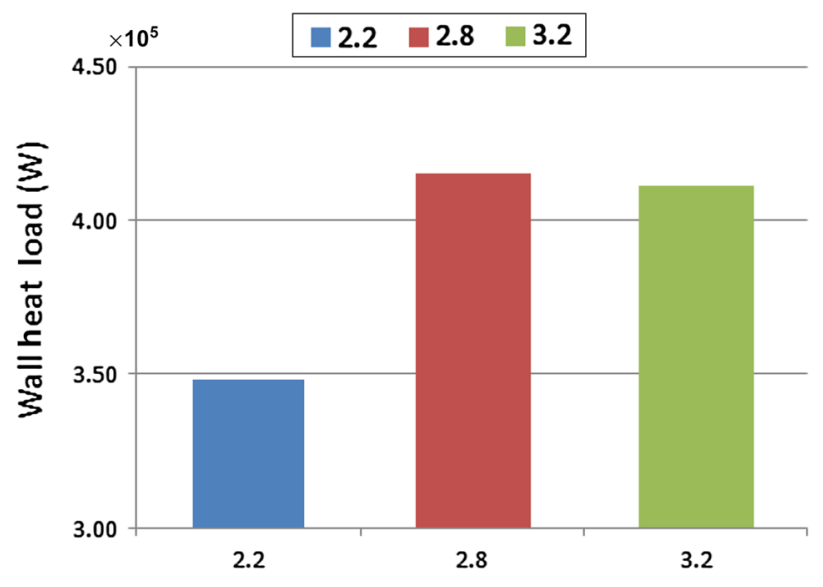

Fig. 23 Total wall heat loading in different cases $(M a=2.2, M a=2.8$, $M a=3.2)$

The contours and the distribution curves of wall heat flux shown in Figs. 20 and 21 indicate that the wall heat flux distribution has a significant difference from cases in which the inlet Mach number is 2.2 and 2.8. There are two local high-heat-flux regions: (1) on the backward face of the cavity and close to the center of the combustor and (2) on the bottom wall near the injection holes. The maximum of wall heat flux is on the backward face of the cavity with a value of $4.4 \mathrm{MW} / \mathrm{m}^{2}$.

\section{Comparison of wall heat flux between the three cases}

Figure 22 gives the statistical diagram of the wall heat loaded on different walls in different cases, and Fig. 23 shows the total wall heat loading in different cases. Some conclusions can be gotten from Figs. 22 and 23. Firstly, as shown in Fig. 22, the bottom wall takes up more than $50 \%$ of the total 
heat loading for all the cases since the fuel/air mixing and combustion occurs mainly near the bottom wall. The wall heat loaded on the top wall rises with the increase of the inlet Mach number, due to that the wall heat on the top wall being only caused by the aerodynamic heating of the supersonic air flow. However, the wall heat values on the bottom wall in different cases are almost the same since the aerodynamic heating and release of combustion heat affect the bottom wall heat flux at the same time. As the inlet Mach number increases, the aerodynamic heating is increased, but the release of combustion heat is decreased due to less amount of fuel at the same fuel-to-air equivalence ratio and higher inlet Mach number. Figure 23 indicates that the change of total wall heat loading is not monotonic with the increase of inlet Mach number; the maximum appears in the case in which the inlet Mach number is 2.8 .

\section{Conclusions}

Flow and heat transfer of a supersonic model combustor are studied via the Favre-averaged Navier-Stokes method with an SST $k-\omega$ turbulence model and a three-step reaction model of ethylene. The present work focuses on the effect of variable inlet Mach numbers on the distributions of wall heat flux and locations of heat flux peaks. Based on the numerical results, a few conclusions can be obtained.

1. When the inlet Mach numbers are 2.2 and 2.8, on account of heat release and pressure rise of combustion, there is a shock train structure in the isolator upstream of the injections. The interaction between shock structures and boundary layer leads to the boundary layer separation in the isolator and near the side wall, which enhances the mixing of fuel and air, which thus enhances the local combustion and results in the local peak of wall heat flux. Meanwhile, the vortex structure that appears in the cavity facilitates mixing and combustion of fuel and air; thus, another peak of wall heat flux is located on the backward face of the cavity.

2. As the inlet Mach number increased to 3.2, no shock structure is observed in the isolator, and the combustor flow is in a typical supersonic combustion mode. As a result, no peak of wall heat flux is identified on the side wall, and the main region of large wall heat flux is the backward face of the cavity.

3. The distributions of local high-heat-flux regions are different in those three cases as well as the locations and values of wall heat flux peaks. By the three-dimensional simulation, it is obvious that the combustion mainly takes place near the bottom wall where the fuel is injected; thus, the heat loading on the bottom wall is largest. The heat flux on the side wall reduces remarkably with the increase of vertical distance, and it is possible that the peak of wall heat flux appears on the side wall due to local flow separation at medium inlet Mach number. The heat flux on the top wall is the lowest one. Thus, the distributions of total temperature and wall heat flux are distinctly non-uniform along the circumferential direction of the combustor.

4. The statistical results show that the bottom wall takes up more than $50 \%$ of the total heat loading and its amount changes very slightly with the inlet Mach number. The change of total wall heat loading is not monotonic with the increase of inlet Mach number, and the maximum appears in the case that inlet Mach number is 2.8 .

Acknowledgements This work was supported by the National Natural Science Foundation of China (Grant 11672307) and the Youth Innovation Promotion Association, Chinese Academy of Sciences.

\section{References}

1. Tsuru, T., Tomioka, S., Murakami, et al.: Skin-friction measurements in supersonic combustion flows of a scramjet combustor. In: 44th AIAA/ASME/SAE/ASEE Joint Propulsion Conference and Exhibit. AIAA 2008-4578, Jul 21-23 (2008)

2. Sturtevant, B., Sanderson, S.R.: Transient heat flux measurement using a surface junction thermocouple. Rev. Sci. Instrum. Acc. 73, 2781-2787 (2002)

3. Cheng, L.W., Zhong, F.Q., Gu, H.B., et al.: Application of conjugate gradient method for measurements of wall heat flux of supersonic combustor. Int. J. Heat. Mass. Tran. Acc. 96, 249-255 (2016)

4. Sun, B., Liu, D., Wang, T.P.: A method to measure wall heat flux of a liquid rocket engine combustion chamber. J. Propul. Technol. Acc. 38, 2122-2129 (2017)

5. Wang, H.B., Wang, Z.G., Sun, M.B., et al.: Large eddy simulation of a hydrogen-fueled scramjet combustor with dual cavity. Acta Astronaut. 108, 119-128 (2015)

6. Ai, Q., Xia, X.L., Guo, L.: Numerical Prediction of radiative heat flux to the combustor wall of a scramjet. J. Eng. Therm. Acc. 28, 112-114 (2017)

7. Jiang, J.P., Li, M., Jin, P., et al.: Simulation and analysis of wall heat fluxes for shear coaxial $\mathrm{GH}_{2} / \mathrm{GO}_{2}$ single element combustor. J. Aerosp. Power Acc. 27, 1881-1886 (2012)

8. Wang, H.B., Wang, Z.G., Sun, M.B., et al.: Combustion modes of hydrogen jet combustion in a cavity-based supersonic combustor. Int. J. Hydrog. Energy 38, 12078-12089 (2013)

9. Lv, Y., Ihme, M.: High-order discontinuous Galerkin method for applications to multicomponent and chemically reacting flows. Acta Mech. Sin. 33, 486-499 (2017)

10. Zhao, W.G., Zheng, H.W., Liu, F.J., et al.: An efficient unstructured WENO method for supersonic reactive flows. Acta Mech. Sin. 34, 623-631 (2018)

11. Wang, X., Zhong, F.Q., Gu, H.B., et al.: Numerical study of combustion and convective heat transfer of a Mach 2.5 supersonic combustor. Appl. Therm. Eng. Acc. 89, 883-896 (2015)

12. Menter, F.R.: Two-equation eddy-viscosity turbulence models for engineering applications. AIAA J. Acc. 32, 1598-1605 (1994)

13. Liou, M.S., Steffen Jr., C.J.: A new flux splitting scheme. J. Comput. Phys. Acc. 107, 23-39 (1993)

14. Westbrook, C.K., Dryer, F.L.: Simplified reaction mechanisms for the oxidation of hydrocarbon fuels in flames. Combust. Sci. Technol. 27, 14-27 (1981) 
15. Magnussen, B.F., Hjertageria, B.H.: On mathematical models of turbulent combustion with special emphasis on soot formation and combustion. Sump. Int. Combust. Acc. 16, 719-729 (1977)

16. Li, J., Ma, F.H., Yang, V., et al: A comprehensive study of ignition transient in an ethylene-fueled scramjet combustor. In: 43rd AIAA/ASME/SAE/ASEE Joint Propulsion Conference and Exhibit. AIAA 2007-5025, July 8-11 (2007)

17. Eckert, E.R.G.: Engineering relations for heat transfer and friction in high velocity laminar and turbulent boundary layer flow over surfaces with constant pressure and temperature. Trans. ASME. Acc. 78, 1273-1283 (1956)
18. Heiser, W.H., Pratt, D.T.: Hypersonic Airbreathing Propulsion. American Institute of Aeronautics and Astronautics Inc, Washington DC (1994)

19. Anderson, J.D.: Hypersonic and High Temperature Gas Dynamics. McGraw-Hill, New York (1989)

20. Micka, D.J., Driscoll, J.F.: Combustion characteristics of a dualmode scramjet combustor with cavity flameholder. Proc. Combust. Inst. Acc. 32, 2397-2404 (2009) 